\title{
АСИМПТОТИКА МАГНЕТОСОПРОТИВЛЕНИЯ
}

\section{А.А. Ериов, Ю.А. Крутова}

Найдена асимптотическая формула, выражающая электрическое сопротивление кристалла изотропного полупроводника в магнитном поле и подключенного с помощью малых контактов. Для моделирования электрического потенциала использовалось решение краевой задачи для эллиптического уравнения с косой производной на границе.

Ключевые слова: магнетосопротивление, потенциал электрического поля, электрическое сопротивление, магнитное поле, асимптотическое разложение, уравнение Лапласа, косая производная, краевая задача.

\section{ОБРАЗЕЦ ЦИТИРОВАНИЯ}

Ершов А.А., Крутова Ю.А. Асимптотика магнетосопротивления // Вестник ЮУрГУ. Серия: Вычислительная математика и информатика. 2016. Т. 5, № 1. С. 5-12. DOI: $10.14529 / \mathrm{cmse} 160101$.

\section{Введение}

Магнетосопротивление (магниторезистивный эффект) - это изменение электрического сопротивления материала в магнитном поле [1]. Математические модели, позволяющие рассчитать магнетосопротивление, зависят от свойств материала и типа проводимости. Мы будем использовать математическую модель, приведенную Н.Н. Поляковым в работе [2] для моделирования электрического потенциала образца изотропного полупроводника прямоугольной формы при протекании через него электрического тока в магнитном поле. В этой работе были проведены эксперименты по следующей схеме (рис. 1).

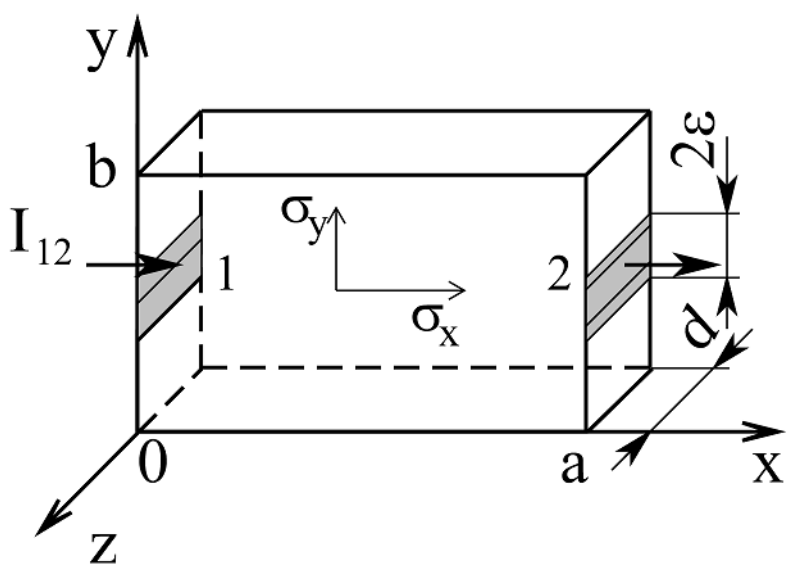

Рис. 1. Схема эксперимента

Образец прямоугольной формы вырезан параллельно кристаллографическим плоскостям из монокристалла арсенида кадмия. По торцам образца расположены контакты 1 и 2, через которые пропускается ток $I_{12}$. Кроме того, весь образец помещен в магнитное поле с индукцией $B_{z}$, которое направлено перпендикулярно плоскости образца. Данная схема применяется в стандартной методике Ван дер Поля по измерению коэффициента Холла анизотропных кристаллов и пленок прямоугольной формы. При этом 
для моделирования электрического потенциала $\phi(x, y)$ при постоянном токе $I_{12}$ и в установившемся режиме Н.Н. Поляковым использовалось решение следующей краевой задачи.

$$
\begin{aligned}
& \frac{\partial^{2} \phi}{\partial x^{2}}+\frac{\sigma_{y}}{\sigma_{x}} \cdot \frac{\partial^{2} \phi}{\partial y^{2}}=0, \quad 0<x<a, 0<y<b, 0<z<d, \\
& \left\{\left.\left(\frac{\partial \phi}{\partial x}+\gamma y \frac{\partial \phi}{\partial y}\right)\right|_{x=0, a}=\left\{\begin{array}{l}
-\frac{I_{12}}{\sigma_{x} 2 \varepsilon d}, \quad y \in\left(\frac{b}{2}-\varepsilon, \frac{b}{2}+\varepsilon\right) \\
0, \quad y \in\left(b, \frac{b}{2}-\varepsilon\right) \cup\left(\frac{b}{2}+\varepsilon, b\right)
\end{array}\right.\right. \\
& \left.\left(\frac{\partial \phi}{\partial y}-\gamma_{x} \frac{\partial \phi}{\partial x}\right)\right|_{y=0, a}=0
\end{aligned}
$$

где $\gamma_{x}=\sigma_{x} R_{z} B_{z}, \gamma_{y}=\sigma_{y} R_{z} B_{z} ; I_{12}$ - ток, протекающий через токовые контакты 1 и 2 ; $\sigma_{x}, \sigma_{y}$ - компоненты тензора электропроводимости; $a, b, d-$ длина, ширина и толщина образца; $2 \varepsilon$ - ширина токовых контактов; $R_{z}$ - компонента тензора Холла при условии, что вектор плотности тока лежит в плоскости $x 0 y$, и поперечное магнитное поле с индукцией $B_{z}$ направлено вдоль оси $0_{z}$.

Решение данной краевой задачи рассматривается нами в классе функций, бесконечно дифференцируемых внутри области и непрерывных вплоть до границы области. В работе Н.Н. Полякова [2] указано, что решение данной задачи методом Фурье в комплексной форме довольно громоздко (и в то же время явный вид решения не приведен), поэтому мы приведем другое, более простое решение.

Отметим, что данная модель применима при достаточно слабом магнитном поле и малых контактах, поскольку, например, плотность тока по сечению контакта не является равномерной, а имеет довольно сложный характер (см., например, [3]).

Нашей задачей является отыскание асимптотики электрического сопротивления прямоугольного образца в магнитном поле при $\varepsilon \rightarrow 0$.

Также отметим, что в случае отсутствия магнитного поля задача эффективного вычисления электрического сопротивления образца с малыми контактами рассматривалась различными методами в двумерном случае в работах [2, 4-6], в трехмерном случае в работах [7-10] и многих других.

В разделе 1 рассмотрен метод Фурье для краевой задачи с косой производной, которым получено решение задачи (1). Раздел 2 содержит вычисление самого магнетосопротивления как функционала от решения задачи и его асимптотику. В заключении подводятся итого проведенного исследования и обсуждаются направления будущих работ.

\section{1. Решение краевой задачи}

Решим краевую задачу (1) с помощью метода Фурье. Обозначим через $\gamma=\sqrt{\frac{\sigma_{y}}{\sigma_{x}}}$. Тогда $\gamma_{y}=\gamma^{2} \gamma_{x}$. 
Будем вначале искать решение в виде произведения $\phi(x, y)=X(x) Y(y)$. Подставим его в уравнение

$$
X^{\prime \prime}(x) Y(y)+\gamma^{2} X(x) Y^{\prime \prime}(y)=0
$$

и разделим переменные

$$
\frac{X^{\prime \prime}(x)}{X(x)}=-\frac{\gamma^{2} Y^{\prime \prime}(y)}{Y(y)}=\lambda .
$$

Здесь $\lambda$ - некоторая неопределенная пока постоянная. Подставим $\phi(x, y)=X(x) Y(y)$ в граничные условия задачи (1):

$$
X(x) Y^{\prime}(0)-\gamma_{x} X^{\prime}(x) Y(0)=0 .
$$

Отсюда получаем, что

$$
X^{\prime}(x)=\mu X(x),
$$

где $\mu=\frac{Y^{\prime}(0)}{\gamma_{x} Y(0)}-$ некоторая постоянная.

Таким образом, одновременно выполняется

$$
\left\{\begin{array}{l}
X^{\prime \prime}(x)=\lambda X(x) \\
X^{\prime}(x)=\mu X(x)
\end{array}\right.
$$

Следовательно,

$$
\left\{\begin{array}{l}
X^{\prime \prime}(x)=\frac{\lambda}{\mu} X^{\prime}(x), \\
X^{\prime \prime}(x)=\mu X^{\prime}(x) .
\end{array}\right.
$$

Отсюда,

$$
\lambda=\mu^{2} .
$$

Аналогичными действиями получим следующую задачу Штурма-Лиувилля для $Y(y)$ :

$$
\left\{\begin{array}{l}
Y^{\prime \prime}(y)=-\frac{\mu^{2}}{\gamma^{2}} Y(y), \\
Y^{\prime}(0)=\gamma_{x} \mu Y(0), \\
Y^{\prime}(b)=\gamma_{x} \mu Y(b) .
\end{array}\right.
$$

Ненулевыми решениями задачи (2) являются

$$
Y_{n}(y)=\cos \left(\frac{\pi n}{b} y+A\right), \quad n \in \mathrm{Z},
$$

где $A=-\operatorname{arctg}\left(\gamma_{x} \gamma\right)$.

Соответственно, функции

$$
X_{n}(x)=e^{\gamma \frac{\pi n}{b} x}, \quad n \in \mathrm{Z}
$$

Общее решение задачи (1) можно искать в виде

$$
\begin{gathered}
\phi(x, y)=B_{0}+A_{0}\left(x+\gamma_{x} y\right)+ \\
+\sum_{n=1}^{\infty} A_{n}\left(e^{\gamma \frac{\pi n}{b} x} \cos \left(\frac{\pi n}{b} y+A\right)+B_{n} e^{-\gamma \frac{\pi n}{b} x} \cos \left(\frac{\pi n}{b} y-A\right)\right) .
\end{gathered}
$$

Поскольку $\gamma_{y}=\gamma^{2} \gamma_{x}, \sin A=-\gamma_{x} \gamma \cos A$, то

$$
\frac{\partial \phi}{\partial x}+\gamma_{y} \frac{\partial \phi}{\partial y}=A_{0}\left(1+\gamma_{x} \gamma_{y}\right)+
$$




$$
+\sum_{n=1}^{\infty} A_{n} \gamma \frac{\pi n}{b}\left(\left(1+\gamma_{x} \gamma_{y}\right) e^{\gamma \frac{\pi n}{b} x}+B_{n}\left(1-\gamma_{x} \gamma_{y}\right) e^{-\gamma \frac{\pi n}{b} x}\right) \cos \left(\frac{\pi n}{b} y\right) \cos A .
$$

Для того чтобы выполнялось равенство

$$
\left.\left(\frac{\partial \phi}{\partial x}+\gamma_{y} \frac{\partial \phi}{\partial y}\right)\right|_{x=0}=\left.\left(\frac{\partial \phi}{\partial x}+\gamma_{y} \frac{\partial \phi}{\partial y}\right)\right|_{x=a}
$$

положим $B_{n}=\frac{1+\gamma_{x} \gamma_{y}}{1-\gamma_{x} \gamma_{y}} e^{\gamma \frac{\pi n}{b} a}$. Тогда

$$
\begin{gathered}
\left.\left(\frac{\partial \phi}{\partial x}+\gamma_{y} \frac{\partial \phi}{\partial y}\right)\right|_{x=0}=\left.\left(\frac{\partial \phi}{\partial x}+\gamma_{y} \frac{\partial \phi}{\partial y}\right)\right|_{x=a}= \\
=A_{0}\left(1+\gamma_{x} \gamma_{y}\right)+\sum_{n=1}^{\infty} A_{n} \gamma \frac{\pi n}{b} \frac{1+\gamma_{x} \gamma_{y}}{\sqrt{1+\gamma_{x}^{2} \gamma_{y}^{2}}}\left(1+e^{\gamma \frac{\pi n}{b} a}\right) \cos \left(\frac{\pi n}{b} y\right),
\end{gathered}
$$

учитывая, что $\cos A=\frac{1}{\sqrt{1+\operatorname{tg}^{2} A}}=\frac{1}{\sqrt{1+\gamma_{x}^{2} \gamma_{y}^{2}}}$.

Обозначим $C_{0}=A_{0}\left(1+\gamma_{x} \gamma_{y}\right), C_{n}=A_{n} \gamma \frac{\pi n}{b} \frac{1+\gamma_{x} \gamma_{y}}{\sqrt{1+\gamma_{x}^{2} \gamma_{y}^{2}}}\left(1+e^{\frac{\gamma \frac{\pi n}{b} a}{b}}\right)$. Тогда на отрезке $y \in[0, b]$ будем иметь равенство

$$
C_{0}+\sum_{n=1}^{\infty} C_{n} \cos \left(\frac{\pi n}{b} y\right)=\left\{\begin{array}{l}
-\frac{I_{12}}{\sigma_{x} 2 \varepsilon d}, \quad y \in\left(\frac{b}{2}-\varepsilon, \frac{b}{2}+\varepsilon\right), \\
0, \quad y \in\left[0, \frac{b}{2}-\varepsilon\right) \cup\left(\frac{b}{2}+\varepsilon, b\right] .
\end{array}\right.
$$

Отсюда,

$$
\begin{gathered}
C_{0}=-\frac{I_{12}}{\sigma_{x} b d} \\
C_{n}=-\frac{I_{12}}{\sigma_{x} 2 \varepsilon d} \cdot \frac{2}{b} \int_{b / 2-\varepsilon}^{b / 2+\varepsilon} \cos \left(\frac{\pi n}{b} y\right) d y=-\frac{I_{12}}{\sigma_{x} 2 \varepsilon d} \cdot \frac{4}{\pi n} \cos \left(\frac{\pi n}{2}\right) \sin \left(\frac{\pi n}{b} \varepsilon\right) .
\end{gathered}
$$

Поскольку $\cos (\pi m)=(-1)^{m}$, то

$$
\begin{gathered}
C_{2 m}=-\frac{I_{12}}{\sigma_{x} \varepsilon d} \cdot \frac{(-1)^{m}}{\pi m} \sin \left(\frac{2 \pi m}{b} \varepsilon\right) . \\
C_{2 m+1}=0 .
\end{gathered}
$$

Таким образом,

$$
\begin{gathered}
A_{0}=-\frac{I_{12}}{\sigma_{x} b d} \cdot \frac{1}{1+\gamma_{x} \gamma_{y}}, \\
A_{2 m}=-\frac{I_{12}}{\sigma_{x} \varepsilon d} \cdot \frac{(-1)^{m} b}{2 \gamma \pi^{2} m^{2}} \cdot \frac{\sqrt{1+\gamma_{x}^{2} \gamma_{y}^{2}}}{1+\gamma_{x} \gamma_{y}} \cdot \frac{\sin \left(\frac{2 \pi m}{b} \varepsilon\right)}{1+e^{\gamma \frac{2 \pi m}{b} a}}, \\
A_{2 m+1}=0 .
\end{gathered}
$$

И окончательно получаем, что

$$
\phi(x, y)=B_{0}-\frac{I_{12}}{\sigma_{x} b d} \cdot \frac{1}{1+\gamma_{x} \gamma_{y}}\left(x+\gamma_{x} y\right)-
$$




$$
\begin{gathered}
-\frac{I_{12}}{\sigma_{x} \varepsilon d} \frac{\sqrt{1+\gamma_{x}^{2} \gamma_{y}^{2}}}{1+\gamma_{x} \gamma_{y}} \sum_{n=1}^{\infty} \frac{(-1)^{m} b}{2 \gamma \pi^{2} m^{2}} \cdot \frac{\sin \left(\frac{2 \pi m}{b} \varepsilon\right)}{1+e^{\gamma \frac{2 \pi m}{b} a}}\left(e^{\gamma \frac{2 \pi m}{b} x} \cos \left(\frac{2 \pi m}{b} y+A\right)+.\right. \\
\left.+\frac{1+\gamma_{x} \gamma_{y}}{1-\gamma_{x} \gamma_{y}} e^{\gamma \frac{2 \pi m}{b}(a-x)} \cos \left(\frac{\pi n}{b} y-A\right)\right) .
\end{gathered}
$$

где $A=-\operatorname{arctg}\left(\gamma_{x} \gamma\right), B_{0}-$ произвольная аддитивная постоянная.

Отметим, что полученный ряд равномерно сходится при $0 \leq x \leq a$ и $0 \leq y \leq b$, а каждое его слагаемое удовлетворяет уравнению из краевой задачи (1). Отсюда следует, что мы действительно построили решение краевой задачи (1) в классе функций

$$
\left.C^{\infty}(\{(x, y): 0<x<a, 0<y<b\}) \cap C(\{x, y\}: 0 \leq x \leq a, 0 \leq y \leq b\}\right) .
$$

\section{2. Вычисление асимптотики магнетосопротивления}

Используя полученное нами решение (3), вычислим величину выделяемой мощности по формуле

$$
\begin{gathered}
W=d \cdot \int_{0}^{a} \int_{0}^{b}\left(\left(\frac{\partial \phi}{\partial x}\right)^{2} \sigma_{x}+\left(\frac{\partial \phi}{\partial y}\right)^{2} \sigma_{y}\right) d y d x= \\
=\frac{a I_{12}^{2}}{\sigma_{x} b d} \frac{1}{1+\gamma_{x} \gamma_{y}}+\frac{2 I_{12}^{2}}{d \pi \sqrt{\sigma_{x} \sigma_{y}}}\left(\frac{1+\gamma_{x}^{2} \gamma_{y}^{2}}{1-\gamma_{x}^{2} \gamma_{y}^{2}}\right)^{2} \sum_{m=1}^{\infty} \frac{\sin ^{2}\left(\frac{2 \pi m}{b} \varepsilon\right) \operatorname{th}\left(\gamma \frac{\pi m}{b} a\right)}{m} .
\end{gathered}
$$

Из последнего равенства легко получить выражение для электрического сопротивления прямоугольной пластины в магнитном поле:

$$
\begin{gathered}
R=\frac{W}{I_{12}^{2}}=\frac{a}{\sigma_{x} b d} \frac{1}{1+\gamma_{x} \gamma_{y}}+ \\
+\frac{2 I_{12}^{2}}{d \pi \sqrt{\sigma_{x} \sigma_{y}}}\left(\frac{1+\gamma_{x}^{2} \gamma_{y}^{2}}{1-\gamma_{x}^{2} \gamma_{y}^{2}}\right)^{2} \sum_{m=1}^{\infty} \frac{\sin ^{2}\left(\frac{2 \pi m}{b} \varepsilon\right)}{\left(\frac{2 \pi m}{b} \varepsilon\right)^{2}} \frac{\operatorname{th}\left(\gamma \frac{\pi m}{b} a\right)}{m} .
\end{gathered}
$$

Заметим, что ряд (4) имеет сложную зависимость от малого параметра $\varepsilon$ : при стремлении $\varepsilon$ к нулю он расходится как гармонический. Однако, в работах [4] и [6] методом замены ряда на интеграл $([11$, гл.2], $[12$, гл.4, §11]) уже была получена полная асимптотика суммы следующего ряда при $\mu \rightarrow 0$ :

$$
\begin{gathered}
S(\mu)=\sum_{n=1}^{\infty} \frac{\sin ^{2}(\mu n)}{(\mu n)^{2}} \frac{\operatorname{th}(\lambda n)}{n}=\ln \frac{1}{2 \mu}+\frac{3}{2}-\sum_{n=1}^{\infty} \frac{e^{-\lambda n}}{n \cdot \operatorname{ch}(\lambda n)}+ \\
+\left(\frac{1}{36}+\frac{1}{3} \sum_{n=1}^{\infty} \frac{n e^{-\lambda n}}{\operatorname{ch}(\lambda n)}\right) \mu^{2}+\left(\frac{1}{2700}-\frac{2}{45} \sum_{n=1}^{\infty} \frac{n^{3} e^{-\lambda n}}{\operatorname{ch}(\lambda n)}\right) \mu^{4}+O\left(\mu^{6}\right) .
\end{gathered}
$$

Таким образом,

$$
R=\frac{a}{\sigma_{x} b d} \frac{1}{1+\gamma_{x} \gamma_{y}}+
$$




$$
+\frac{2}{d \pi \sqrt{\sigma_{x} \sigma_{y}}}\left(\frac{1+\gamma_{x}^{2} \gamma_{y}^{2}}{1-\gamma_{x}^{2} \gamma_{y}^{2}}\right)^{2}\left(\ln \frac{e^{3 / 2} b}{4 \pi \varepsilon}-\sum_{n=1}^{\infty} \frac{e^{-\frac{\pi a}{b} \gamma m}}{n \cdot \operatorname{ch}\left(\frac{\pi a}{b} \gamma m\right)}\right)+O\left(\frac{\varepsilon^{2}}{b^{2}}\right) .
$$

\section{Заключение}

В статье рассмотрены сложности моделирования магниторезистивного эффекта. Решение приведенной задачи (1) и соответствующий функционал, выражающий магнетосопротивление, могут быть найдены численно. Однако, в таком случае его зависимость от параметров остается неясной. В данной работе мы выписали явное решение с помощью метода Фурье, а затем вычислили по нему электрическое сопротивление в виде суммы довольно сложного ряда. Опираясь на недавно полученные результаты, нам удалось найти асимптотику его суммы, в результате чего получена простая асимптотическая формула, наглядно показывающая зависимость электрического сопротивления проводника от величины напряженности магнитного поля и сечения контактов. Используя данную формулу, нетрудно вычислить и величину магнетосопротивления, которая является изменением электрического сопротивления под воздействием магнитного поля. Отметим, что при увеличении напряженности магнитного поля электрическое сопротивление растет из-за главного члена асимптотики $\frac{2}{d \pi \sqrt{\sigma_{x} \sigma_{y}}}\left(\frac{1+\gamma_{x}^{2} \gamma_{y}^{2}}{1-\gamma_{x}^{2} \gamma_{y}^{2}}\right)^{2} \ln \frac{1}{\varepsilon}$, что и наблюдается в большинстве случаев за редким исключением так называемого отрицательного магнетосопротивления.

В дальнейшем было бы интересно найти такие конфигурации проводника и контактов, при которых бы магнетосопротивление было бы отрицательным, или показать, что такое невозможно в данной модели.

Работа выполнена при поддержке гранта РНФ (проект 15-11-10018).

\section{Литература}

1. Киреев П.С. Физика полупроводников. М.: Высшая школа. Изд-во 2, доп, 1975. $584 \mathrm{c.}$

2. Поляков Н.Н. Об измерении коэффициент Холла электропроводимости анизотропных проводников // Заводская лаборатория. 1989. № 3. С. 20-22.

3. Гришенцев А.Ю. Моделирование распределения плотности тока в сложном неоднородном проводнике. Часть $1 / /$ Научно-технический вестник СПбГУ ИТМО. 2006. № 29. C. 87-94.

4. Ершов А.А. Краевая эллиптическая задача с дельтообразной производной на границе // Журнал вычислительной математики и математической физики. 2010. Т. 50. № 3. C. $479-485$.

5. Ершов А.А. К задаче об измерении электропроводности // Журнал вычислительной математики и математической физики. 2013. Т. 53. № 6. С. 1004-1007.

6. Ершов А.А. Асимптотика решения уравнения Лапласа со смешанными условиями на границе // Журнал вычислительной математики и математической физики. 2011. T. 51. № 7. С. 1064-1080.

7. Хольм Р. Электрические контакты. М.: Иностранная литература, 1961. 314 с. 
8. Павлейно О.М., Павлов В.А., Павлейно М.А. Уточнение границ применимости хольмовского приближения для расчета сопротивления электрических контактов // Электронная обработка материалов. 2010. Т. 46. № 5. С. 56-62.

9. Затовский В.Г., Минаков Н.В. Экспериментальное моделирование сопротивления стягивания // Электрические контакты и электроды. 2010. № 10. С. 132-139.

10. Филиппов В.В., Поляков Н.Н. Распределения потенциала в анизотропных проводниковых кристаллах и пленки при измерении электропроводимости и коэффициента Холла // Вести высших учебных заведений черноземья. 2011. № 2(24). С. 6-10.

11. Ильин А.М., Данилин А.Р. Асимптотические методы в анализе. М.: Физматлит, 2009. $222 \mathrm{c}$.

12. Федорюк М.В. Асимптотика, интегралы и ряды. М.: Наука, 1987. 544 с.

Ершов Александр Анатольевич, к.ф.-м.н., научный сотрудник отдела динамических систем, Институт математики и механики имени Н.Н. Красовского Уральского отделения РАН (Екатеринбург, Российская Федерация), ale10919@yandex.ru.

Крутова Юлия Александровна, студент математического факультета, Челябинский государственный университет (Челябинск, Российская Федерация), lulia_74rus@mail.ru.

Поступила в редакцию 4 июля 2015 г.

Bulletin of the South Ural State University Series "Computational Mathematics and Software Engineering" 2016, vol. 5, no. 1, pp. 5-12

DOI: $10.14529 / \mathrm{cmse160101}$

\section{ASIMPTOTICS OF MAGNETORESISTANCE}

A.A. Ershov, Institute of Mathematics and Mechanics, Ural branch of RAS, Ekaterinburg, Russian Federation

J.A. Krutova, Chelyabinsk State University, Chelyabinsk, Russian Federation

An asymptotic formula expressing the electric the resistance of the isotropic crystal semiconductor in a magnetic field and connected through small contacts was found. For modeling of the electric potential the solution of boundary value problems for elliptic equations with oblique derivative on the boundary was used.

Keywords: magnetoresistance, electric potential, electrical resistance, magnetic field, asymptotic expansion, Laplace equation, oblique derivative, boundary value problem.

\section{FOR CITATION}

Ershov A.A., Krutova J.A. Asimptotics of Magnetoresistance. Bulletin of the South Ural State University. Series: Computational Mathematics and Software Engineering. 2016. vol. 5, no. 1. pp. 5-12. (in Russian) DOI: 10.14529/cmse160101.

\section{References}

1. Kireev P.S. Fizika poluprovodnikov [Physics of semiconductors]. Moscow, Higher school, 1975. 584 p. (in Russian) 
2. Polyakov N.N. Ob izmerenii koeffitsient Kholla elektroprovodimosti anizotropnykh provodnikov [The measurement of Hall coefficient of conductivity of anisotropic conductors]. Zavodskaya laboratoriya [Factory laboratory]. 1989. no. 3. pp. 20-22. (in Russian)

3. Grishentsev A.Yu. Modelirovanie raspredeleniya plotnosti toka v slozhnom neodnorodnom provodnike. Chast' 1 [Simulation of current density distribution in complex inhomogeneous conductor. Part 1]. Nauchno-tekhnicheskiy vestnik SPbGU ITMO [Scientific and technical Bulletin of SPbSU ITMO]. 2006. no. 29. pp. 87-94. (in Russian)

4. Ershov A.A. Asymptotics of the solution to the Neumann problem with a delta-functionlike boundary function. Computational Mathematics and Mathematical Physics. 2010. vol. 50, no. 3. pp. 457-463. DOI: 10.1134/S0965542510030073.

5. Ershov A.A. On measurement of electrical conductivity // Computational Mathematics and Mathematical Physics. 2013. vol. 53. no. 6. pp. 823-826. DOI: 10.1134/S0965542513060079.

6. Ershov A.A. Asymptotics of the solution of Laplace's equation with mixed boundary conditions. Computational Mathematics and Mathematical Physics. 2011. vol. 51, no. 7. pp. 994-1010. DOI: 10.1134/S0965542511060066.

7. Holm R. Electric contacts handbook. Berlin, Springer-Verlag Berlin Heidelberg GmbH, 1958. 522 p.

8. Pavleyno O.M., Pavlov V.A., Pavleyno M.A. Verification of the Boundaries of the Applicabillity of the Holm Approximation for the Calculation of the Resistance of Electric Contacts. Surface Engineering and Applied Electrochemistry. 2010. vol. 46, no. 5. pp. $440-446$.

9. Zatovskiy V.G., Minakov N.V. Eksperimental'noe modelirovanie soprotivleniya styagivaniya [Experimental simulation of the resistance of the tightening]. Elektricheskie kontakty $i$ elektrody [Electrical contacts and electrodes]. 2010. no. 10. pp. 132-139. (in Russian)

10. Filippov V.V., Polyakov N.N. Raspredeleniya potentsiala $\mathrm{v}$ anizotropnykh provodnikovykh kristallakh i plenki pri izmerenii elektroprovodimosti i koeffitsienta Kholla [The potential distribution in the anisotropic conductive crystals and films in the measurement of conductivity and Hall coefficient]. Vesti vysshikh uchebnykh zavedeniy chernozem'ya [News of higher educational institutions of Chernozem region]. 2011. no. 2(24). pp. 6-10. (in Russian)

11. Il'in A.M., Danilin A.R. Asimptoticheskie metody $v$ analize [Asymptotic methods in analysis]. M.: Fizmatlit, 2009. 222 p. (in Russian)

12. Fedoryuk M.V. Asimptotika, integraly $i$ ryady [Asymptotics, integrals and series]. M.: Nauka, 1987. 544 p. (in Russian)

Received July 4, 2015. 